\title{
Diet quality and telomere length in older Australian men and women
}

\author{
Catherine M. Milte $^{1} \cdot$ Aaron P. Russell $^{1} \cdot$ Kylie Ball $^{1} \cdot$ David Crawford $^{1} \cdot$ Jo Salmon $^{1} \cdot$ \\ Sarah A. McNaughton ${ }^{1}$
}

Received: 11 April 2016 / Accepted: 10 October 2016 / Published online: 26 October 2016

(C) The Author(s) 2016. This article is published with open access at Springerlink.com

\begin{abstract}
Purpose Telomere length is a biomarker of cellular ageing, with longer telomeres associated with longevity and reduced risk of chronic disease in older age. Consumption of a healthy diet may contribute to longevity via its impact on cellular ageing, but studies on diet and telomere length to date have been limited and their findings equivocal. The aim of this study was to examine associations between three indices of diet quality and telomere length in older men and women.

Methods Adults aged 57-68 years participating in the Wellbeing, Eating and Exercise for a Long Life (WELL) study in Victoria, Australia $(n=679)$, completed a postal survey including an 111-item food frequency questionnaire in 2012. Diet quality was assessed via three indices: the Dietary Guideline Index, the Recommended Food Score, and the Mediterranean Diet Score. Relative telomere length was measured by quantitative polymerase chain reaction. Associations between diet quality and telomere length were assessed using linear regression adjusted for covariates.

Results After adjustment for age, sex, education, smoking, physical activity, and body mass index (BMI), there were no significant associations between diet quality and relative telomere length.
\end{abstract}

Electronic supplementary material The online version of this article (doi:10.1007/s00394-016-1326-6) contains supplementary material, which is available to authorized users.

Catherine M. Milte

catherine.milte@deakin.edu.au

1 Institute for Physical Activity and Nutrition (IPAN), School of Exercise and Nutrition Sciences, Deakin University, Geelong, Australia
Conclusions In a sample of older adults residing in Victoria, Australia, men and women aged 57-68 years with better-quality diets did not have longer telomeres. Further investigation in longitudinal studies will determine whether diet can influence telomere length over time in an ageing population.

Keywords Diet · Ageing · Telomere length · Diet quality · Mediterranean diet

\section{Introduction}

The world's ageing population continues to increase with the number of persons aged 60 years and over expected to exceed the number of children in the world by 2045 [1]. Increased longevity is supporting marked growth in the proportion of adults aged over 85 years [2]. As chronic disease burden increases with age, it is important that health and function are maintained to complement increased longevity. Diet plays a key role in maintaining health in adulthood and may impact on markers of cellular ageing such as telomere length.

Telomeres are repetitive DNA sequences at the ends of eukaryotic chromosomes that undergo attrition each time a somatic cell divides [3]. They prevent loss of genomic DNA at ends of linear chromosomes and protect their physical integrity with cell division and replication, thereby protecting against cell death. Telomere length decreases with age and is considered a biomarker of accelerated cellular ageing. Shorter telomere length is associated with decreased life expectancy and increased risk of chronic disease [4] and was recently described as one of the nine "hallmarks of ageing" [5]. As telomere attrition rate varies between individuals and is increased by inflammation and 
oxidative stress [4], it is a proposed modifiable lifestyle risk factor for health and longevity in older age. Although there is some genetic regulation of variability of telomere length, it may also be partially explained by lifestyle behaviours, including dietary intake [6]. The intake of various individual nutrients including folate, vitamins $\mathrm{C}, \mathrm{E}, \mathrm{D}, \mathrm{A}$, and $\beta$-carotene, magnesium and omega- 3 polyunsaturated fatty acids is associated with longer telomere length [6, 7]. However, there has been little investigation into the association between whole diet, or dietary patterns and telomere length.

Whilst previous research into nutrition and health in older age has focussed on the role of individual nutrients or foods, there is increasing interest in dietary pattern analysis as a chronic disease determinant [8]. Dietary patterns can be defined by two approaches: multivariate statistical techniques such as factor or cluster analysis (data-driven approaches), and dietary scoring methods informed by a priori guidelines and recommendations, or diet quality indices. Diet quality indices can assess adherence to dietary guidelines [9], or a particular type of diet such as the Mediterranean diet [10]. Diet quality assessed by adherence to dietary guidelines has been associated with cardiometabolic risk factors [11], and physical and mental health [12, 13], whilst diet quality assessed by adherence to a Mediterranean diet and higher dietary variety has been associated with reduced risk of mortality [14] in older people.

Despite the wide variety of diet quality indices available, few studies have investigated dietary patterns and telomere length in older people $[15,16]$, and only one has included multiple indices of diet quality [17]. Considering the limited research to date, assessing multiple indices of diet quality may give greater insights into which components or aspects of diet quality have greatest influence on telomere length. The aim of this study was to examine associations between three measures of diet quality and relative telomere length in men and women aged 57 years and over.

\section{Methods}

\section{Design}

This study is based on data from the Wellbeing, Eating and Exercise for a Long Life (WELL) study. The WELL study is a prospective, population-based longitudinal cohort study of nutrition and physical activity behaviours, obesity and quality of life, and the intrapersonal, social and environmental influences on these among mid-aged and older adults [18]. Participants aged between 55 and 65 years, living independently in the community and able to complete a written questionnaire in English, were included. In 2010, participants aged between 55 and 65 years, living in the community in urban or rural Victoria, Australia, were selected from the Australian Electoral Roll, stratified by socioeconomic position using the Socioeconomic Index for Areas score (SEIFA) [19]. Potential participants living in a suburb with a population of less than 1000 overall or $<200$ in the 55-65 years age bracket were excluded. All eligible suburbs were classified by SEIFA and divided into tertiles (representing low, medium, and high SEIFA). Fourteen postcodes from each SEIFA tertile were randomly selected. From each postcode, 134 participants (equal numbers of men and women) were selected for invitation into the study. A total of 11,256 surveys were distributed to potential participants at baseline in 2010. Of these, 380 were returned as undeliverable and 95 were returned from individuals outside the age bracket. In total, 4082 completed surveys were returned at baseline (response rate 38\%). Participation was voluntary, and informed consent was obtained by return of the survey. In 2012, participants who agreed to take part in a follow-up were sent a similar survey $(n=3368)$. Of these, 2758 completed surveys were returned (response rate $82 \%$ ). Data were collected at the same time of year in 2010 and 2012 to negate any potential seasonal effects. Ethical approval for the study was obtained from the Deakin University Human Research Ethics Committee (2009-105). Full details of the study have been described elsewhere [18].

In 2012, fasting blood samples were collected in a subgroup of volunteers as part of the WELL Heart Health sub-study. Volunteers invited to take part in this subgroup needed to be dwelling in suburbs classified as urban or urban fringe at the time of the 2012 survey (to allow access to pathology centres where blood samples were taken). A sample of $n=1283$ (667 women and 616 men) were invited to participate via an invitation pack sent out to their postal address, with 884 consenting to take part $(69 \%$ response rate) and 777 providing a blood sample. A total of 768 whole blood samples were collected in EDTA-coated tubes, and telomere length was successfully measured in 764 samples.

\section{Telomere length}

Relative telomere length was measured in whole blood by quantitative polymerase chain reaction (qPCR) [20]. All assays were processed by the same technician under identical conditions. DNA was extracted using the QIAamp DNA blood mini kit (cat \# 51106; Qiagen, Clifton Hill, VIC) according to the manufacturer's protocol.

Relative telomere length was determined using $\mathrm{qPCR}$ to compare telomere repeat sequence copy number $(T)$ to a single-copy gene copy number $(S)(T / S)$, as previously described $[20,21]$. The primers for the telomere qPCR were tel-c 5'-CGGTTTGTTTGGGTTTGGGTTTGGGTT 
TGGGTTTGGGTT-3', and tel-g 5'-GGCTTGCCTTACC CTTACCCTTACCCTTACCCTTACCCT- ${ }^{\prime}$, and were used at final concentrations of 100 and $900 \mathrm{nM}$, respectively. The primers for the single-copy gene, human beta-globulin, were h- $\beta$ gFor $5^{\prime}$-GCTTCTGACACAACTGTGTTCAC TAGC- $3^{\prime}$ and h- $\beta g R e v 5^{\prime}$-CAC CAA CTT CAT CCA CGT TCA CC- $3^{\prime}$, both used at $300 \mathrm{nM}$. Each $20-\mu \mathrm{l}$ qPCR reaction contained $1 \mathrm{U}$ AmpliTaq Gold 360 DNA polymerase with $1 \times$ AmpliTaq Gold 360 buffer (cat \# 4398892; Life Technologies), $2 \mathrm{mM} \mathrm{MgCl}, 1 \mathrm{mM}$ dithiothreitol (cat \# 43186; Sigma-Aldrich, St Louis, MO, USA), 1 M betaine (cat \# B0300-5VL; Sigma-Aldrich), $0.2 \mathrm{mM}$ dNTP mix (cat \# 18427-088 1; Life Technologies), 0.01 mM SYTO 9 Green Fluorescent Nucleic Acid Stain (cat \# S34854; Life Technologies), $5 \mathrm{ng}$ DNA, and the primers of interest. A seven-point standard curve was generated using serial dilutions from 40 to $0.625 \mathrm{ng}$ of DNA from a pooled sample. Aliquots were prepared for each of the serial dilutions so that the same samples were used for standard curve on each PCR plate without freeze thawing. The standard curves for telomere and beta-globulin were both included on each PCR plate. Each standard and sample was measured in duplicate in 384-well plates (cat \# HSP-3801, Bio-Rad, Hercules, CA, USA) using the Bio-Rad C1000 thermal cycler. Cycling conditions consisted of one cycle at $95{ }^{\circ} \mathrm{C}$ for $10 \mathrm{~min}$, followed by 40 cycles at $95^{\circ} \mathrm{C}$ for $5 \mathrm{~s}, 60{ }^{\circ} \mathrm{C}$ for $20 \mathrm{~s}$, and $72{ }^{\circ} \mathrm{C}$ for $60 \mathrm{~s}$. A dissociation curve was generated to show that only one product had been amplified during the PCR.

To determine the relative telomere length, the telomere and human beta-globulin standard curves were plotted as $\log [\mathrm{DNA}]$ concentration against the PCR Ct value. A linear straight line $(y=m x+C)$ was generated for the telomere and beta-globulin standard curves, and the $\log [\mathrm{DNA}]$ concentration for telomere and beta-globulin was determined for each unknown sample. Relative telomere length was expressed as telomere-to-standard single-copy gene ratio ( $T / S$ ratio). The inter-assay coefficient of variation was $3.1 \%$ whilst the intra-assay coefficient of variation was $0.8 \%$.

\section{Dietary intake}

Usual dietary intake at baseline was assessed using an 111item food frequency questionnaire (FFQ) [22, 23], which assessed self-reported intake of food and beverages over the last 6 months. The FFQ has been previously used in other national studies [24-26]. We have also previously used this questionnaire to assess dietary patterns and diet quality and have demonstrated it is a good predictor of health outcomes [9]. The survey included seven additional validated short questions on food habits including salt use (during and after cooking), type of milk and bread consumed, trimming the fat from meat and daily fruit and vegetable consumption $[26,27]$. Frequencies were converted into daily equivalents for analysis [28].

\section{Diet quality}

Diet quality was assessed using three previously developed indices: the Dietary Guideline Index (DGI), the Recommended Food Score (RFS), and the Mediterranean Diet Score (MDS). The indices were adapted for use with the data from the FFQ. The DGI is an updated version of a previous index developed to reflect Australian guidelines for optimal eating patterns which was shown to be a good measure of diet quality [9]. The index was updated to reflect the 2013 Australian Dietary Guidelines [29]. For each dietary guideline component, indicators from the FFQ were identified and food groupings determined. Age- and sex-specific scoring cut-offs for the five core food groups (vegetables, fruits, grains, meat and alternatives, and dairy), fluids, and discretionary foods were devised. Discretionary foods (also commonly known as "extra" foods) are foods that are not essential to provide nutrient requirements due to the high content of sugar, fat, and salt such as soft drinks, cordials, fruit juice drinks, chips, confectionary, chocolate, hamburgers, meat pies, pizza, cakes and muffins, pies and pastries, biscuits, and alcoholic beverages [29]. Items referring to whole-grain cereals, lean protein, reduced/low-fat dairy, unsaturated fats, and dietary variety were included in the index. A total of 13 components were included in the updated DGI. Each component of the DGI was scored proportionally from 0 to 10 , where 10 indicated that a participant was fully meeting the recommendation. The total score was the sum of 13 items so that the diet score had a possible range of $0-130$, with higher scores reflecting greater compliance with the Australian Dietary Guidelines. The previous version of the DGI was evaluated in the Australian population and shown to be related in predicted directions to sociodemographic factors, health behaviours, self-assessed health, and intakes of key nutrients [9, 13].

The RFS is a food-based score which assesses the frequency of consumption of a range of foods considered to be consistent with existing dietary guidelines [30]. The RFS has been previously validated and is associated with biomarkers of dietary intake, chronic disease, and mortality [30, 31]. Participants are allocated a score of 1 for each recommended food consumed more than once per week. A total of 49 foods listed in the FFQ were considered as recommended (consistent with dietary guidelines: fruit, vegetable, whole grain, lean meat and alternatives, and low-fat dairy groups) resulting in scores ranging from 0 to 49 , with higher scores associated with a wider variety of consumption of recommended foods and greater diet quality. 
Adherence to a Mediterranean diet was assessed using the most commonly used MDS developed by Trichopoulou et al. [10]. This index has demonstrated validity through reported relationships with nutrient biomarkers [32] and health outcomes [14, 33-35]. The MDS includes vegetables (excluding potatoes), legumes, fruits and nuts, cereals, fish and seafood, dairy products, meat and meat products, and alcohol. In accordance with standard scoring techniques, we calculated sex-specific medians for frequency of intake of these items. For vegetables, legumes, fruits and nuts, cereals, fish and seafood, participants with an intake above the median were assigned a score of 1 . For dairy products and meat and meat products, participants with an intake below the median were assigned a score of 1 . For alcohol, a score of 1 was assigned for low to moderate intake (intake of no more than 2 times/day) and a score of 0 for no alcohol intake or intake $>2$ times per day. Supplementary Table S1 (Online Resource 1) shows the median frequency of intake for the eight food items included in the MDS. Total score was calculated as the sum of all components, and ranged from 0 to 8 , with higher scores reflecting greater adherence to a Mediterranean diet. An olive oil consumption or monounsaturated-to-saturated fat ratio item is also usually included. We were unable to include this item as it was not assessed in the FFQ. This adapted version of the MDS was previously shown to be associated with health-related quality of life in this cohort [13].

\section{Covariates}

Participant characteristics were collected during the postal survey, including age, country of birth, marital status, retirement status, and smoking status. Menopausal status was collected in women. Highest education level achieved was collected as a measure of socioeconomic position. Self-reported height and weight were collected for calculation of body mass index (BMI; $\mathrm{kg} / \mathrm{m}^{2}$ ). Total physical activity was captured using the self-administered International Physical Activity Questionnaire (IPAQ-L), which has demonstrated validity and reliability in a 12-country, 14-site study [36]. The IPAQ-L assesses duration, frequency, and intensity of leisure, work, commuting, and household/yard activities during the past 7 days. Responses were converted into total metabolic equivalent of task (MET) hours per week with moderate physical activity at $3 \mathrm{MET}$ and vigorous physical activity at $6 \mathrm{MET}$.

\section{Statistical analysis}

Participants missing $>10 \%$ responses on the FFQ, 1 or more responses to the dietary habits questions, or the $T / S$ ratio or covariates were excluded from analysis. Differences between included and excluded participants were explored using $t$ tests for normally distributed variables, the Wilcoxon rank sum test (Mann-Whitney test) for nonnormally distributed variables, and Chi-square tests for categorical variables. Summary of participant characteristics was presented by quartile of relative telomere length. For the main analysis, associations between diet quality and relative telomere length were assessed via linear regression with relative telomere length as a continuous variable. Covariates adjusted for in regression models were selected based on previous literature [9, 37-39], and only those associated with the outcome or exposure were included in the models. Multivariable linear regression models were adjusted for age, sex, and education (model 1), with further adjustments made for smoking, total physical activity (model 2) and BMI (model 3). BMI was included in the final adjustment as obesity may attenuate associations between diet quality and health outcomes, and so the results of analyses with (model 3) and without BMI (model 2) could be assessed separately. StataSE version 13.1 (StataCorp, TX, USA) was used for all statistical analyses. $P<0.05$ was considered significant.

\section{Results}

There was complete data available for analysis on 679 participants (Table 1). Characteristics of participants included in the analysis and participants with incomplete data not included in the analysis are summarised in supplementary Table S2 (Online Resource 2). The age of participants included in analysis ranged from 57 to 68 years. Women had a longer relative telomere length than men (mean $\pm \mathrm{SD} ; 1.01 \pm 0.14$ vs. $0.97 \pm 0.14, P<0.01$, respectively). Relative telomere length did not differ by age, smoking status, BMI, or physical activity.

Table 2 shows the association between diet quality assessed by the DGI-2013, RFS and MDS, and relative telomere length. There were no associations between diet quality and relative telomere length.

\section{Discussion}

In this study of 679 community-dwelling older men and women from the WELL study, greater diet quality assessed via the DGI-2013, RFS, and MDS was not cross-sectionally associated with longer relative telomere length. Previous studies have reported cross-sectional associations between higher intakes of vegetables and lower intakes of saturated fat and sugar-sweetened soda and longer telomere length [40, 41]. Reported associations between diet quality and telomere length have been limited, with only one study investigating associations between multiple dietary indices 
Table 1 Characteristics of 679 men and women from the WELL Study by quartile of relative telomere length, Victoria, Australia, 2012

\begin{tabular}{|c|c|c|c|c|c|c|}
\hline & Total & $Q 1$ & $Q 2$ & & $Q 3$ & $Q 4$ \\
\hline$T / S$ ratio, mean (SD) & $0.99(0.14)$ & $0.82(0.08)$ & 0.9 & $.03)$ & $1.04(0.03)$ & $1.17(0.07)$ \\
\hline$T / S$ ratio, range & $0.43-1.4$ & $0.43-0.89$ & 0.8 & & $0.99-1.09$ & $1.09-1.4$ \\
\hline DGI-2013, mean (SD) & $88.3(13.0)$ & $86.6(12.9)$ & 88. & 3.3) & $88.6(12.4)$ & $90.1(13.2)$ \\
\hline RFS, mean (SD) & $24.7(7.91)$ & $24.5(8.15)$ & 24. & $.50)$ & $24.4(8.02)$ & $25.2(8.00)$ \\
\hline \multirow[t]{2}{*}{ MDS, mean (SD) } & $4.25(1.56)$ & $4.28(1.52)$ & 4.3 & $.64)$ & $4.04(1.60)$ & $4.34(1.45)$ \\
\hline & Mean (SD) & Mean (SD) & Mean (SD) & Mean (SD) & Mean (SD) & $P$ for trend \\
\hline Age & $62.7(3.07)$ & $63.0(3.14)$ & $62.9(2.97)$ & $62.4(3.03)$ & $62.6(3.14)$ & 0.507 \\
\hline BMI & $26.7(4.94)$ & $27.4(5.49)$ & $25.9(4.30)$ & $26.8(4.65)$ & $26.8(5.16)$ & 0.116 \\
\hline \multirow[t]{2}{*}{ Total physical activity ${ }^{\mathrm{a}}$} & $97.2(84.2)$ & $99.5(86.6)$ & $97.8(79.9)$ & $105(96.7)$ & $86.4(71.1)$ & 0.204 \\
\hline & $n(\%)$ & $n(\%)$ & $n(\%)$ & $n(\%)$ & $n(\%)$ & \\
\hline Sex, men & $330(49)$ & $95(56)$ & $81(48)$ & $82(48)$ & $64(38)$ & 0.011 \\
\hline Smoking & & & & & & 0.542 \\
\hline Never smoked & $396(58.3)$ & $95(55.1)$ & $92(54.1)$ & $105(61.8)$ & $104(61.5)$ & \\
\hline Former smoker & $228(33.6)$ & $57(33.5)$ & $64(37.7)$ & $55(32.4)$ & $52(30.8)$ & \\
\hline Daily smoker & $55(8.10)$ & $18(10.6)$ & $14(8.24)$ & $10(5.88)$ & $13(7.69)$ & \\
\hline Country of birth ${ }^{b}$ & & & & & & 0.559 \\
\hline Australia & $515(80.0)$ & $131(77.1)$ & $133(78.2)$ & $131(77.1)$ & $120(71.4)$ & \\
\hline UK & $54(7.96)$ & $16(9.41)$ & $10(5.88)$ & $14(8.24)$ & $14(8.33)$ & \\
\hline Other & $109(16.1)$ & $23(13.5)$ & $27(15.9)$ & $25(14.7)$ & $34(20.2)$ & \\
\hline Marital status $^{\mathrm{b}}$ & & & & & & 0.604 \\
\hline Married/defacto & $520(76.8)$ & $131(77.1)$ & $124(73.8)$ & $136(80.0)$ & $129(76.3)$ & \\
\hline Separated/divorced & 79 (11.7) & $22(12.9)$ & $22(13.1)$ & $18(10.6)$ & $17(10.1)$ & \\
\hline Widowed & $38(5.61)$ & $8(4.71)$ & $11(6.54)$ & $9(5.29)$ & $10(5.92)$ & \\
\hline Never married & $40(5.90)$ & $9(5.29)$ & $11(6.54)$ & $7(4.12)$ & $13(7.69)$ & \\
\hline Education & & & & & & 0.011 \\
\hline Up to 10 years & $165(24.3)$ & $45(26.5)$ & $40(23.5)$ & $43(25.3)$ & 37 (21.9) & \\
\hline 12 years/trade/certificate & $232(34.2)$ & $66(38.8)$ & $68(40.0)$ & $56(32.9)$ & $42(24.8)$ & \\
\hline University degree & $282(41.5)$ & $59(34.7)$ & $62(36.5)$ & $71(41.8)$ & $90(53.3)$ & \\
\hline
\end{tabular}

$B M I$ body mass index, DGI Dietary Guideline Index, MDS Mediterranean Diet Score, RFS Recommended Food Score, T/S ratio relative telomere length, WELL Wellbeing, Eating and Exercise for a Long Life

a Reported as MET hours per week

${ }^{\mathrm{b}}$ Reduced sample size for variable due to missing data

Table 2 Multivariable-adjusted regression coefficients and 95\% CI per unit increase in diet quality score for relative telomere length (T/S ratio) ( $n=679)$

\begin{tabular}{|c|c|c|c|c|c|c|}
\hline \multirow[t]{2}{*}{ Telomere length } & \multicolumn{2}{|l|}{ DGI $(n=679)$} & \multicolumn{2}{|l|}{$\operatorname{MDS}(n=679)$} & \multicolumn{2}{|l|}{$\operatorname{RFS}(n=679)$} \\
\hline & $\beta(95 \% \mathrm{CI})$ & $P$ value & $\beta(95 \% \mathrm{CI})$ & $P$ value & $\beta(95 \% \mathrm{CI})$ & $P$ value \\
\hline Model 1 & $0.001(-0.000,0.001)$ & 0.17 & $-0.001(-0.008,0.006)$ & 0.77 & $-0.0004(-0.002,0.001)$ & 0.60 \\
\hline Model 2 & $0.001(-0.000,0.001)$ & 0.17 & $-0.001(-0.008,0.006)$ & 0.79 & $-0.0004(-0.002,0.001)$ & 0.61 \\
\hline Model 3 & $0.001(-0.000,0.001)$ & 0.17 & $-0.001(-0.008,0.006)$ & 0.82 & $-0.0004(-0.002,0.001)$ & 0.62 \\
\hline
\end{tabular}

DGI Dietary Guideline Index, MDS Mediterranean Diet Score, RFS Recommended Food Score, BMI body mass index

Model 1: controlling for age, sex, and education, Model 2: model $1+$ smoking and total physical activity, Model 3: model $2+$ BMI 
and telomere length. In 4676 women, aged $42-70$ years from the Nurses' Health Study, higher scores on the Mediterranean diet and Alternate Healthy Eating Index were associated with longer telomere length. However, no associations between prudent and Western dietary patterns and telomere length were observed [17]. A cross-sectional study of 217 men and women aged 71-87 years reported that a greater adherence to a Mediterranean diet was associated with longer leucocyte telomere length and higher telomerase activity, a relationship moderated by inflammation [15]. Following a 5-year Mediterranean diet intervention, an association between TL and changes in adiposity indices was observed in 521 men and women aged 55-80 years [42]. Furthermore, in the same cohort, telomere shortening appeared to be attenuated with greater adherence to the Mediterranean diet in people carrying the Pro12Ala polymorphism in the peroxisome proliferator-activated receptor gamma 2 (PPARy2) gene [43].

Studies investigating dietary patterns and telomere length longitudinally have been limited to date. A randomised crossover intervention of 20 older adults found reduced telomere shortening following a Mediterranean diet for 4 weeks compared to a saturated fatty acid diet and low-fat high-carbohydrate diet [44]. More recently, a randomised intervention trial of the Mediterranean diet in 520 participants aged 55-80 years reported greater adherence to a Mediterranean diet was associated longer telomeres in women only at baseline [45]. However, no beneficial effects for the Mediterranean diet on telomere length were observed at 5-year follow-up, and a higher risk of telomere shortening was observed in the Mediterranean diet and nuts group.

Associations between inflammatory dietary patterns derived by principal component analysis and leucocyte telomere length in 840 men and women aged $45-84$ years have been investigated [16]. No relationship between telomere length and a "fats and processed meat" pattern or a "whole grains and fruit" pattern was observed, despite these patterns being associated with C-reactive protein, interleukin-6, and homocysteine [16]. However, an association between intake of processed meat and shorter telomeres was reported. A recent Mediterranean diet intervention study of 520 participants at high cardiovascular risk reported a pro-inflammatory diet characterised by a dietary inflammatory index was associated with shorter telomeres cross-sectionally and after a 5-year follow-up [46].

Previous studies have also investigated associations between healthy lifestyle and telomere length. In 612 patients with advanced prostate cancer and 1049 matched controls, the sum score of a healthier lifestyle and diet factors (low or no cigarette use, higher fruit and vegetable intake, lower BMI, more physical activity), correlated with longer telomere length; no correlations between telomere length were observed when these factors were analysed individually [47]. In patients with low-risk prostate cancer, a comprehensive lifestyle intervention (changes to diet, physical activity, stress management, and social support) resulted in increased telomere length and telomerase activity, when comparing baseline and 5-year follow-up values. Adherence to the lifestyle changes was also significantly associated with relative telomere length when adjusted for age and length of follow-up [48]. Similarly, in a cohort of 5862 women (aged mean $58.7 \pm 0.09$ years), increased telomere length was associated with adherence to a healthy lifestyle; the later defined using five components including smoking, physical activity, adiposity, alcohol use, and diet [49].

Previous studies have reported inverse correlations between age and telomere length [15-17, 50, 51]. However, no association was observed between age and telomere length in the current study. The age range of our sample of 57-68 years was considerably narrower than that of previous studies, with reported age ranges of 42-70 [17], 71-87 [15], 45-84 [16], 1-96 [50], and 18-90 [51] years. In the present study, the $T / S$ values ranged between 0.43 and 1.40 which are similar to values observed previously within the same age range [50]. Our lack of observation of an association between age and telomere length is most likely due to the small age range. This is not without precedence and there appears to be limited correlation between age and telomere length when analysed within narrow age ranges of 40-44 and 60-64 years [52].

Diet quality has been shown to be associated with mortality [14] and reduced risk of chronic diseases such as cardiovascular disease and diabetes [53], all outcomes that have been associated with shortening of telomeres [4]. Previously, it has been proposed that consumption of a healthier diet would protect against age-related telomere loss through reduced oxidative stress and inflammation [6]. The DGI was associated with lower intakes of energy, total fat and saturated fat, and higher intakes of fibre, $\beta$-carotene, vitamin $\mathrm{C}$, folate, calcium, and iron previously [9]. The RFS was associated with intake of similar nutrients and antioxidants and also reduced markers of chronic disease and inflammation, including serum homocysteine, C-reactive protein, plasma glucose, total serum cholesterol, and blood pressure in previous research [31]. The Mediterranean diet is also proposed to reduce disease risk through its anti-inflammatory and anti-oxidative properties [54]. In summary, although previous research demonstrates the potential for all three indices used in the current study to assess long-term adherence to diets associated with reduced inflammation and oxidative stress, no significant associations with telomere length were observed.

These findings are generalisable to community-dwelling men and women around the "peri-retirement" stage. 
However, it should be noted that WELL study participants reported higher scores on the RAND 36-item survey [13] compared to other Australian population-based samples $[55,56]$, and may therefore represent a group with better health status than the Australian population. The samples are also not reflective of the oldest old or specific clinical populations, and therefore, these findings may not be applicable across the older age range in a broader sense. However, there are challenges to assessing telomere length in older age, as the upper age limit for reliable assessment of telomere length is 75 years [57]. Telomere length instability and "survivor bias", where people who live longer are suspected to possess longer telomeres and some resilience to chronic disease, may contribute to a lack of association between telomere length and mortality observed above this age $[57,58]$. However, this is unlikely to have played a role in the lack of associations observed in the current study.

The strengths of our study include the well-characterised sample, detailed assessment of dietary intake and potential confounders and the use of three validated diet quality indices. However, the study has some limitations that should be considered. Only a subsample of the original cohort provided a blood sample, which may have created a selection bias. Dietary intake was assessed by a non-quantified FFQ, which did not allow for adjustment of energy intake in statistical analysis. However, key determinants of energy intake were taken into account by adjusting for sex, BMI, and physical activity. Although a range of potential covariates were tested for inclusion in the regression models, it is possible some residual confounding remained. Finally, the relatively modest sample size may have limited our ability to detect the small associations reported by previous studies, although this is unlikely since some of those studies were conducted in a sample of similar size to the current study $[15,17]$.

A potential limitation of studies investigating the Mediterranean diet in non-Mediterranean samples is the lack of foods and eating behaviours truly reflective of a traditional Mediterranean diet. However, studies in Australian [59, 60] and UK samples [61] have reported the presence of a Mediterranean-style pattern. Our choice of Mediterranean Diet Index included sample-specific median intakes of vegetables, legumes, fruits and nuts, cereals, and fish as cut points for scoring. Although this enables adaptation of the score for a non-Mediterranean population, it likely reflects a dietary pattern with substantially lower absolute intakes of these foods compared to typical of Mediterranean populations as supported by a recent Australian study [62]. An olive oil or monounsaturated fat component was not included in the MDS in this study due to limitations in the FFQ. However, some controversy exists regarding the inclusion of this item in Mediterranean diet assessment in non-Mediterranean populations, as olive oil consumption is typically low and monounsaturated-to-saturated fat ratio instead reflects animal fat intake, which is low in the typical Mediterranean diet [63].

Although telomere length is thought to vary depending on exposure to environmental factors, telomere length is also determined by genetics [64] and shows inter-individual variation from birth [65]. Thus, attempts to determine the association between diet and telomere attrition should ideally measure within-person change in telomere length over long periods of time. The current study was cross-sectional and was therefore unable to assess within-person change in telomere length. This may have limited the ability to detect significant associations in the current study. It should be noted that all previous studies investigating dietary patterns and telomere length have also been cross-sectional. Therefore, inconsistencies in the evidence to date including the current study may be related to the fact that telomere length shows inter-individual variation and telomere length at any age relates to a combination of telomere length at birth and subsequent rate of change [66]. Considering the variation in findings of this and previous studies, it is critical that future studies are longitudinal in study design and include assessment of telomere length over time to determine a true measure of biological ageing.

\section{Conclusion}

In a sample of adults residing in Victoria, Australia, men and women aged 57-68 years with better-quality diets did not have longer telomeres. Further investigation of telomere length over time in longitudinal studies will determine whether dietary patterns are associated with cellular ageing in an older population.

Funding This project was awarded funding from the Diabetes Australia Research Trust and the Australian Research Council (ARC) (ID: DP1095595, FT100100581). KB was supported by a Principal Research Fellowship from the Australian National Health \& Medical Research Council (NHMRC), ID 1042442. SAM was supported by an NHMRC Career Development Fellowship Level 2, ID1104636 and was previously supported by an ARC Future Fellowship (20112015, FT100100581). JS was supported by a NHMRC Principal Research Fellowship (APP1026216). The contents of this manuscript are the responsibility of the authors and do not reflect the views of the NHMRC. The funding sources had no involvement in the study design, data collection and analysis, writing of the article or decision to submit the article for publication.

\section{Compliance with ethical standards}

Conflict of interest The authors declare that they have no conflict of interest.

Ethical standards The study was approved by the Deakin University Human Research Ethics Committee (2009-105) and has therefore been performed in accordance with the ethical standards laid down in the 
1964 Declaration of Helsinki and its later amendments. Participation was voluntary, and informed consent was obtained by return of the survey.

Open Access This article is distributed under the terms of the Creative Commons Attribution 4.0 International License (http://creativecommons.org/licenses/by/4.0/), which permits unrestricted use, distribution, and reproduction in any medium, provided you give appropriate credit to the original author(s) and the source, provide a link to the Creative Commons license, and indicate if changes were made.

\section{References}

1. United Nations (2009) World population ageing. Economic and Social Affairs Population Division, New York

2. Australian Institute of Health and Welfare (2007) Older Australia at a glance, vol 52, 4th edn. AIHW, Canberra

3. Blackburn EH (1991) Structure and function of telomeres. Nature 350(6319):569-573

4. Sanders JL, Newman AB (2013) Telomere length in epidemiology: a biomarker of aging, age-related disease, both, or neither? Epidemiol rev 35(1):112-131

5. Lopez-Otin C, Blasco MA, Partridge L, Serrano M, Kroemer G (2013) The hallmarks of aging. Cell 153(6):1194-1217

6. Paul L (2011) Diet, nutrition and telomere length. J Nutr Biochem 22(10):895-901

7. O'Callaghan N, Parletta N, Milte CM, Benassi-Evans B, Fenech M, Howe PR (2014) Telomere shortening in elderly individuals with mild cognitive impairment may be attenuated with omega-3 fatty acid supplementation: a randomized controlled pilot study. Nutrition 30(4):489-491

8. Newby PK, Tucker KL (2004) Empirically derived eating patterns using factor or cluster analysis: a review. Nutr Rev 62(5):177-203

9. McNaughton SA, Ball K, Crawford D, Mishra GD (2008) An index of diet and eating patterns is a valid measure of diet quality in an Australian population. J Nutr 138(1):86-93

10. Trichopoulou A, Orfanos P, Norat T, Bueno-de-Mesquita B, Ocke MC, Peeters PH, van der Schouw YT, Boeing H, Hoffmann K, Boffetta P, Nagel G, Masala G, Krogh V, Panico S, Tumino R, Vineis P, Bamia C, Naska A, Benetou V, Ferrari P, Slimani N, Pera G, Martinez-Garcia C, Navarro C, RodriguezBarranco M, Dorronsoro M, Spencer EA, Key TJ, Bingham S, Khaw KT, Kesse E, Clavel-Chapelon F, Boutron-Ruault MC, Berglund G, Wirfalt E, Hallmans G, Johansson I, Tjonneland A, Olsen A, Overvad K, Hundborg HH, Riboli E, Trichopoulos D (2005) Modified Mediterranean diet and survival: EPICelderly prospective cohort study. BMJ 330(7498):991

11. McNaughton SA, Dunstan DW, Ball K, Shaw J, Crawford D (2009) Dietary quality is associated with diabetes and cardiometabolic risk factors. J Nutr 139(4):734-742

12. Woo J, Chan R, Leung J, Wong M (2010) Relative contributions of geographic, socioeconomic, and lifestyle factors to quality of life, frailty, and mortality in elderly. PLoS One 5(1):e8775

13. Milte CM, Thorpe MG, Crawford D, Ball K, McNaughton SA (2015) Associations of diet quality with health-related quality of life in older Australian men and women. Exp Gerontol 64:8-16

14. McNaughton SA, Bates CJ, Mishra GD (2012) Diet quality is associated with all-cause mortality in adults aged 65 years and older. J Nutr 142(2):320-325
15. Boccardi V, Esposito A, Rizzo MR, Marfella R, Barbieri M, Paolisso G (2013) Mediterranean diet, telomere maintenance and health status among elderly. PLoS One 8(4):e62781

16. Nettleton JA, Diez-Roux A, Jenny NS, Fitzpatrick AL, Jacobs DR Jr (2008) Dietary patterns, food groups, and telomere length in the Multi-Ethnic Study of Atherosclerosis (MESA). Am J Clin Nutr 88(5):1405-1412

17. Crous-Bou M, Fung TT, Prescott J, Julin B, Du M, Sun Q, Rexrode KM, Hu FB, De Vivo I (2014) Mediterranean diet and telomere length in Nurses' Health Study: population based cohort study. BMJ 349:g6674

18. McNaughton SA, Crawford D, Ball K, Salmon J (2012) Understanding determinants of nutrition, physical activity and quality of life among older adults: the Wellbeing, Eating and Exercise for a Long Life (WELL) study. Health Qual Life Outcomes 10:109

19. Australian Bureau of Statistics (2003) 2001 Census of population and housing: information paper-socio economic indexes for areas (Cat. No. 2039.0). Commonwealth of Australia, Canberra

20. Cawthon RM (2002) Telomere measurement by quantitative PCR. Nucleic Acids Res 30(10):e47

21. Krauss J, Farzaneh-Far R, Puterman E, Na B, Lin J, Epel E, Blackburn E, Whooley MA (2011) Physical fitness and telomere length in patients with coronary heart disease: findings from the Heart and Soul Study. PLoS One 6(11):e26983

22. Hodge A, Patterson AJ, Brown WJ, Ireland P, Giles G (2000) The Anti Cancer Council of Victoria FFQ: relative validity of nutrient intakes compared with weighed food records in young to middle-aged women in a study of iron supplementation. Aust N Z J Public Health 24(6):576-583

23. Ireland P, Jolley D, Giles G, O'Dea K, Powles J, Rutishauser I, Wahlqvist ML, Williams J (1994) Development of the Melbourne FFQ: a food frequency questionnaire for use in an Australian prospective study involving an ethnically diverse cohort. Asia Pac J Clin Nutr 3:19-23

24. Smith KJ, McNaughton SA, Gall SL, Blizzard L, Dwyer T, Venn AJ (2010) Involvement of young Australian adults in meal preparation: cross-sectional associations with sociodemographic factors and diet quality. J Am Diet Assoc 110(9):1363-1367

25. Smith KJ, Gall SL, McNaughton SA, Blizzard L, Dwyer T, Venn AJ (2010) Skipping breakfast: longitudinal associations with cardiometabolic risk factors in the Childhood Determinants of Adult Health Study. Am J Clin Nutr 92(6):1316-1325

26. McLennan W, Podger A (1995) National nutrition survey users' guide. Australian Bureau of Statistics, Canberra

27. Rutishauser IHE, Webb K, Abraham B, Allsopp R (2001) Evaluation of short dietary questions from the 1995 National Nutrition Survey. National Food and Nutrition Monitoring and Surveillance Project, Canberra

28. Willett W (2013) Nutritional epidemiology, 3rd edn. Oxford University Press, New York

29. National Health and Medical Research Council (2013) Australian dietary guidelines. National Health and Medical Research Council, Canberra

30. Kant AK, Schatzkin A, Graubard BI, Schairer C (2000) A prospective study of diet quality and mortality in women. JAMA 283(16):2109-2115

31. Kant AK, Graubard BI (2005) A comparison of three dietary pattern indexes for predicting biomarkers of diet and disease. J Am Coll Nutr 24(4):294-303

32. Benitez-Arciniega AA, Mendez MA, Baena-Diez JM, Rovira Martori MA, Soler C, Marrugat J, Covas MI, Sanz H, Llopis A, Schroder H (2011) Concurrent and construct validity of Mediterranean diet scores as assessed by an FFQ. Public Health Nutr 14(11):2015-2021 
33. Bollwein J, Diekmann R, Kaiser MJ, Bauer JM, Uter W, Sieber CC, Volkert D (2013) Dietary quality is related to frailty in community-dwelling older adults. J Gerontol A Biol Sci Med Sci 68(4):483-489

34. Milaneschi Y, Bandinelli S, Corsi AM, Lauretani F, Paolisso G, Dominguez LJ, Semba RD, Tanaka T, Abbatecola AM, Talegawkar SA, Guralnik JM, Ferrucci L (2011) Mediterranean diet and mobility decline in older persons. Exp Gerontol 46(4):303-308

35. Bosetti C, Gallus S, Trichopoulou A, Talamini R, Franceschi S, Negri E, La Vecchia C (2003) Influence of the Mediterranean diet on the risk of cancers of the upper aerodigestive tract. Cancer Epidemiol Biomark Prev 12(10):1091-1094

36. Craig CL, Marshall AL, Sjostrom M, Bauman AE, Booth ML, Ainsworth BE, Pratt M, Ekelund U, Yngve A, Sallis JF, Oja P (2003) International physical activity questionnaire: 12-country reliability and validity. Med Sci Sports Exerc 35(8):1381-1395

37. Conklin AI, Maguire ER, Monsivais P (2013) Economic determinants of diet in older adults: systematic review. J Epidemiol Commun Health 67(9):721-727

38. Needham BL, Adler N, Gregorich S, Rehkopf D, Lin J, Blackburn EH, Epel ES (2013) Socioeconomic status, health behavior, and leukocyte telomere length in the National Health and Nutrition Examination Survey, 1999-2002. Soc Sci Med 85:1-8

39. Puterman E, Lin J, Krauss J, Blackburn EH, Epel ES (2015) Determinants of telomere attrition over 1 year in healthy older women: stress and health behaviors matter. Mol Psychiatry 20(4):529-535

40. Tiainen AM, Mannisto S, Blomstedt PA, Moltchanova E, Perala MM, Kaartinen NE, Kajantie E, Kananen L, Hovatta I, Eriksson JG (2012) Leukocyte telomere length and its relation to food and nutrient intake in an elderly population. Eur J Clin Nutr 66(12):1290-1294

41. Leung CW, Laraia BA, Needham BL, Rehkopf DH, Adler NE, Lin J, Blackburn EH, Epel ES (2014) Soda and cell aging: associations between sugar-sweetened beverage consumption and leukocyte telomere length in healthy adults from the National Health and Nutrition Examination Surveys. Am J Public Health 104(12):2425-2431

42. Garcia-Calzon S, Gea A, Razquin C, Corella D, Lamuela-Raventos RM, Martinez JA, Martinez-Gonzalez MA, Zalba G, Marti A (2014) Longitudinal association of telomere length and obesity indices in an intervention study with a Mediterranean diet: the PREDIMED-NAVARRA trial. Int J Obes (Lond) 38(2):177-182

43. Garcia-Calzon S, Martinez-Gonzalez MA, Razquin C, Corella D, Salas-Salvado J, Martinez JA, Zalba G, Marti A (2015) Pro12Ala polymorphism of the PPARgamma2 gene interacts with a mediterranean diet to prevent telomere shortening in the PREDIMED-NAVARRA randomized trial. Circ Cardiovasc Genet 8(1):91-99

44. Marin C, Delgado-Lista J, Ramirez R, Carracedo J, Caballero J, Perez-Martinez P, Gutierrez-Mariscal FM, Garcia-Rios A, Delgado-Casado N, Cruz-Teno C, Yubero-Serrano EM, Tinahones F, Malagon Mdel M, Perez-Jimenez F, Lopez-Miranda J (2012) Mediterranean diet reduces senescence-associated stress in endothelial cells. Age (Dordr) 34(6):1309-1316

45. Garcia-Calzon S, Martinez-Gonzalez MA, Razquin C, Aros F, Lapetra J, Martinez JA, Zalba G, Marti A (2016) Mediterranean diet and telomere length in high cardiovascular risk subjects from the PREDIMED-NAVARRA study. Clin Nutr. doi:10.1016/j. clnu.2016.03.013

46. Garcia-Calzon S, Zalba G, Ruiz-Canela M, Shivappa N, Hebert JR, Martinez JA, Fito M, Gomez-Gracia E, Martinez-Gonzalez MA, Marti A (2015) Dietary inflammatory index and telomere length in subjects with a high cardiovascular disease risk from the PREDIMED-NAVARRA study: cross-sectional and longitudinal analyses over 5 years. Am J Clin Nutr 102(4):897-904

47. Mirabello L, Huang WY, Wong JY, Chatterjee N, Reding D, Crawford ED, De Vivo I, Hayes RB, Savage SA (2009) The association between leukocyte telomere length and cigarette smoking, dietary and physical variables, and risk of prostate cancer. Aging Cell 8(4):405-413

48. Ornish D, Lin J, Chan JM, Epel E, Kemp C, Weidner G, Marlin R, Frenda SJ, Magbanua MJ, Daubenmier J, Estay I, Hills NK, Chainani-Wu N, Carroll PR, Blackburn EH (2013) Effect of comprehensive lifestyle changes on telomerase activity and telomere length in men with biopsy-proven low-risk prostate cancer: 5-year follow-up of a descriptive pilot study. Lancet Oncol 14(11):1112-1120

49. Sun Q, Shi L, Prescott J, Chiuve SE, Hu FB, De VI, Stampfer MJ, Franks PW, Manson JE, Rexrode KM (2012) Healthy lifestyle and leukocyte telomere length in US women. PLoS One 7(5):e38374

50. Hewakapuge S, van Oorschot RA, Lewandowski P, BaindurHudson S (2008) Investigation of telomere lengths measurement by quantitative real-time PCR to predict age. Leg Med (Tokyo) 10(5):236-242

51. Lapham K, Kvale MN, Lin J, Connell S, Croen LA, Dispensa BP, Fang L, Hesselson S, Hoffmann TJ, Iribarren C, Jorgenson E, Kushi LH, Ludwig D, Matsuguchi T, McGuire WB, Miles S, Quesenberry CP Jr, Rowell S, Sadler M, Sakoda LC, Smethurst D, Somkin CP, Van Den Eeden SK, Walter L, Whitmer RA, Kwok PY, Risch N, Schaefer C, Blackburn EH (2015) Automated assay of telomere length measurement and informatics for 100,000 subjects in the genetic epidemiology research on adult health and aging (GERA) cohort. Genetics 200(4):1061-1072

52. Mather KA, Jorm AF, Milburn PJ, Tan X, Easteal S, Christensen $H$ (2010) No associations between telomere length and age-sensitive indicators of physical function in mid and later life. J Gerontol A Biol Sci Med Sci 65(8):792-799

53. Schwingshackl L, Hoffmann G (2015) Diet quality as assessed by the healthy eating index, the alternate healthy eating index, the dietary approaches to stop hypertension score, and health outcomes: a systematic review and meta-analysis of cohort studies. J Acad Nutr Diet 115(5):780-800

54. Marin C, Yubero-Serrano EM, Lopez-Miranda J, Perez-Jimenez F (2013) Endothelial aging associated with oxidative stress can be modulated by a healthy mediterranean diet. Int J Mol Sci 14(5):8869-8889

55. Australian Bureau of Statistics (1997) National Health Survey: SF-36 population norms. Australian Bureau of Statistics, Canberra

56. Butterworth P, Crosier T (2004) The validity of the SF-36 in an Australian National Household Survey: demonstrating the applicability of the household income and labour dynamics in Australia (HILDA) survey to examination of health inequalities. BMC Public Health 4:44

57. Martin-Ruiz CM, Gussekloo J, van Heemst D, von Zglinicki T, Westendorp RG (2005) Telomere length in white blood cells is not associated with morbidity or mortality in the oldest old: a population-based study. Aging Cell 4(6):287-290

58. Cawthon RM, Smith KR, O'Brien E, Sivatchenko A, Kerber RA (2003) Association between telomere length in blood and mortality in people aged 60 years or older. Lancet 361(9355):393-395. doi:10.1016/S0140-6736(03)12384-7

59. Rienks J, Dobson AJ, Mishra GD (2013) Mediterranean dietary pattern and prevalence and incidence of depressive symptoms in mid-aged women: results from a large community-based prospective study. Eur J Clin Nutr 67(1):75-82

60. Mishra GD, McNaughton SA, Ball K, Brown WJ, Giles GG, Dobson AJ (2010) Major dietary patterns of young and middle 
aged women: results from a prospective Australian cohort study. Eur J Clin Nutr 64(10):1125-1133

61. Brunner EJ, Mosdol A, Witte DR, Martikainen P, Stafford M, Shipley MJ, Marmot MG (2008) Dietary patterns and 15-year risks of major coronary events, diabetes, and mortality. Am J Clin Nutr 87(5):1414-1421

62. Crichton GE, Bryan J, Hodgson JM, Murphy KJ (2013) Mediterranean diet adherence and self-reported psychological functioning in an Australian sample. Appetite 70:53-59

63. Flood VM, Webb KL, Rochtchina E, Kelly B, Mitchell P (2007) Fatty acid intakes and food sources in a population of older Australians. Asia Pac J Clin Nutr 16(2):322-330

64. Andrew T, Aviv A, Falchi M, Surdulescu GL, Gardner JP, Lu X, Kimura M, Kato BS, Valdes AM, Spector TD (2006) Mapping genetic loci that determine leukocyte telomere length in a large sample of unselected female sibling pairs. Am J Hum Genet 78(3):480-486

65. Graakjaer J, Pascoe L, Der-Sarkissian H, Thomas G, Kolvraa S, Christensen K, Londono-Vallejo JA (2004) The relative lengths of individual telomeres are defined in the zygote and strictly maintained during life. Aging Cell 3(3):97-102

66. Allison DB, Antoine LH, Ballinger SW, Bamman MM, Biga P, Darley-Usmar VM, Fisher G, Gohlke JM, Halade GV, Hartman JL, Hunter GR, Messina JL, Nagy TR, Plaisance EP, Powell ML, Roth KA, Sandel MW, Schwartz TS, Smith DL, Sweatt JD, Tollefsbol TO, Watts SA, Yang Y, Zhang J, Austad SN (2014) Aging and energetics' 'Top 40' future research opportunities 2010-2013. F1000Research 3:219 